\title{
Development of Physiological Parameters Gateway for Medical IOT Ward
}

\author{
He Shi-Lin ${ }^{1,2,}$, Li Bei $^{2, *}$, Ying Jun², Chen Guang-Fei ${ }^{2}$, Lin Shu-Chao², Zhou Dan² and Yu Meng-Sum ${ }^{1}$ \\ ${ }^{I}$ Institute of Medical Equipment, Academy of Military Medical Science, Tianjin, China \\ ${ }^{2}$ PLA General Hospital, Beijing, China
}

\begin{abstract}
Internet of things (IOT) gateway is an important part of all kinds of IOT applications within the healthcare industry. To meet the needs from wireless transfer to management of physiological parameters in hospital, a kind of IOT gateway for physiological parameters service is designed. Carrier sensing technology, automatic networking technology, and multi-thread programming technology are adopted to design and manufacture the gateway. A hardware gateway and a physiological parameters gateway server with functions of protocol translation, routing management, and data security control are designed to deal with different needs of the sensor network in the Medical IOT Ward. The physiological parameters gateway, which is the key application device in medical IOT ward, is designed to fulfil intelligent collection and comprehensive convergence of daily physiological parameters. The medical IOT ward simplifies the work of physiological parameters collection which is used to be finished manually, and provides reliable security assurance for the patients' daily care. It will have a high value in clinical application.
\end{abstract}

Keywords: Gateway, healthcare, intelligent monitor, IOT, physiological parameters.

\section{INTRODUCTION}

Health care service is in a rapid developing and everchanging era. As in aging society, new medical model is gradually changing the way of disease diagnosis, treatment, prevention and control. A new generation of digital health engineering technology is required to cope with this change. With ubiquitous sensing device interoperability, IOT technology provides support such as; health care information automatic collection, intelligent transmission, comprehensive aggregation, full decision-making analysis and the whole process assistance, which increases the capacity and efficiency of health care services, improve medical services quality and models; and finally fully realize the medical wisdom.

To access the medical IOT, medical IOT gateways are urgently needed to be developed for the identification of all types of medicine resources and state signals of human life. The IOT gateway can offer a rapid secure access to the IOT for all kinds of medical equipment. Medical IOT gateway is a key device in IOT. So it is far more important than the traditional Internet gateway.

The functional design of IOT gateway mainly considers two key factors. On the one hand, it should implement several independent, small-scale and heterogeneous wireless sensors converged into a networking, and make such sensor networking to provide a particular service into a full-service networking, and provide a variety of means for the IOTs to access to Internet. However, the medical IOT gateway design will make it possible to access to the medical services at anytime and anywhere. On the other hand, it is not realistic

*Address correspondence to these authors at the Institute of Medical Equipment, Academy of Military Medical Science, Tianjin, China; E-mail: heshilin301@163.com, libei301@163.com to manage the network assessment by IOT terminals integrated with low-power and low-energy characteristic sensors, and to control a huge number of IOT terminals directly by the server, which will be more complex and consumable. Therefore, application of medical IOT gateway to manage medical equipment network access is optimal choice $[1,2]$.

This paper is to meet with the requirement of patients' physiological parameters collection in the daily ward, including body temperature, blood pressure, pulse, Electrocardiograph (ECG), etc. An overall structure of the medical IOT was described and the data transmission protocol specification was defined. The IOT hardware gateways and IOT devices for physiological parameters collection in inpatient wards are designed to meet the demands of the acquisition, collection and distribution physiological parameters. Furthermore, a medical data forwarding protocol transit routing and data management for IOT are defined. This study will be a valuable reference for the design of physiological parameters collection based on the whole wireless networking system.

\section{SYSTEM CONSTRUCTION}

The new medical IOT ward is armed with physiological parameter acquisition devices, such as wireless electronic thermometers, wireless sphygmomanometer and ECG monitor and so on. The design focuses on main tough problems on private clinical information management, such as how to configure these devices for physiological parameters timely collection and how to get the real-time data of these devices. For example, in PLA General Hospital, there are different wireless systems running simultaneously in a ward, including wireless temperature, pulse and blood pressure acquisition system. Though this device has not been applied widely, but we confronted many problems pertaining to management and maintenance. After doing some research on the data 
structures of different physiological parameters and the applications of the different IOT devices, we designed a two kinds of gateway model, the physiological parameters gateway and the hardware gateway, to cope with these difficulties mentioned above.

The main technical framework for physiological data acquisition system IOT ward is shown in Fig. (1). Body temperature, blood pressure and ECG are the main parameters which are needed for diagnosis every day in the ward. The special equipments and softwares are designed for intelligent acquisition of these parameters including: thermometer, sphygmomanometer, ECG monitor with wireless communication capabilities, the nurses' station computer, the Hospital Information System (HIS) server, the physiological parameters gateway and the hardware gateway. Through the patients' physiological signal acquisition devices and gateways in the ward, the physiological parameters information is transmitted to the hospital's internal network [2, 3]. After confirmed by the nurses, the data is transferred directly to the HIS server.

\section{MEDICAL IOT GATEWAY}

For the complication facing managing various types of perception terminals, the physiological parameters IOT gateway are required to support heterogeneous network interconnection, protocol translation and routing aggregation, address allocation and recovery, data aggregation, and terminal control functions, which provide users easier access to the interface. IOT gateway for networking systems setup a bridge between hospital information network interconnected and monitoring devices, ensuring a rapid transmission, low equipment cost, flexible access, and high reliability.

While a large number of terminal sensors and multiple heterogeneous wireless networks access to the Internet gateway at the same time, there must be a lot of data rush to the hardware gateway and physiological parameters gateway as is shown in the Fig. (1). Direct communication and interaction with sensing terminal through hardware gateway and physiological parameters gateway realize data forwarding, data gathering, data analysis and data security. The management of the data in medical IOT gateway is shown in Fig. (2).

The mechanism including effective device authentication, user authentication business safety and equipment safety management is needed between Hardware gateway and physiological parameters gateway. However, this study only focuses on the hardware gateway protocol definition, hardware gateway anti-collision control in low-power network, real-time high-volume data forwarding and on-line gateway monitoring.

Ward physiological parameters gateway Framework as shown in the Figure 1 above, mainly contains two parts. One is low-power network. It mainly considers the application need, portable and low power consumption demand. The designed gateway can adopt RF radio frequency (RF), infrared, Bluetooth and ZigBee, and connect all kinds of sensors to a private network of low power consumption, like wireless electronic thermometer etc. The other is Hospital internal network. It can receive data from lots of devices and directly access to LAN or WAN, then their data can be sent to the physiological parameter gateway for processing directly. Wireless sphygmomanometer, based on $\mathrm{Wi}-\mathrm{Fi}$, is one kind of the device that can send its data directly to the physiological parameter gateway. Two different gateways mentioned above are used to solve the problem of communication between types of equipment like terminal and platform. As the design of the hardware gateway and the physiological parameters gateway, physiological parameters data can run not only on the wireless sensor, but also on a variety of heterogeneous underlying network so as to realizing the connectivity of all kinds of physical devices and achieving the connectivity of heterogeneous networks [4-7].

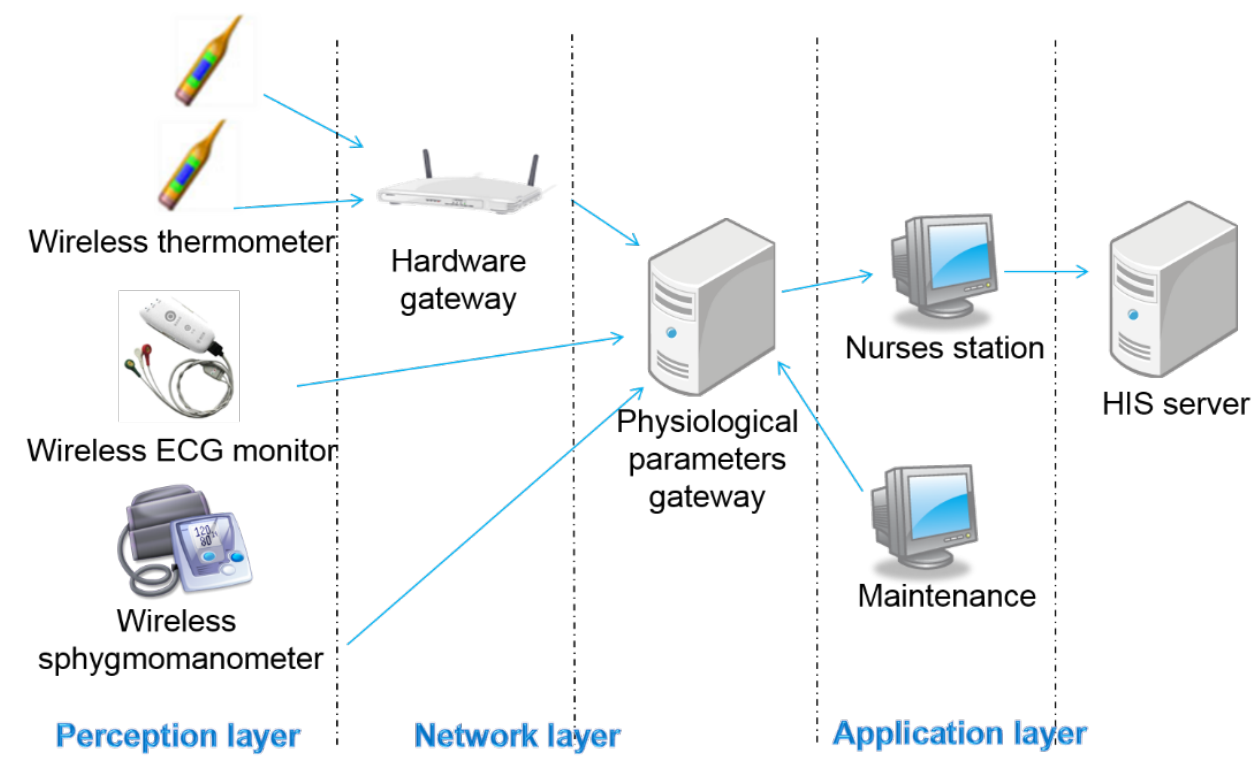

Fig. (1). Framework of physiological parameter acquisition system of medical IOT ward. 


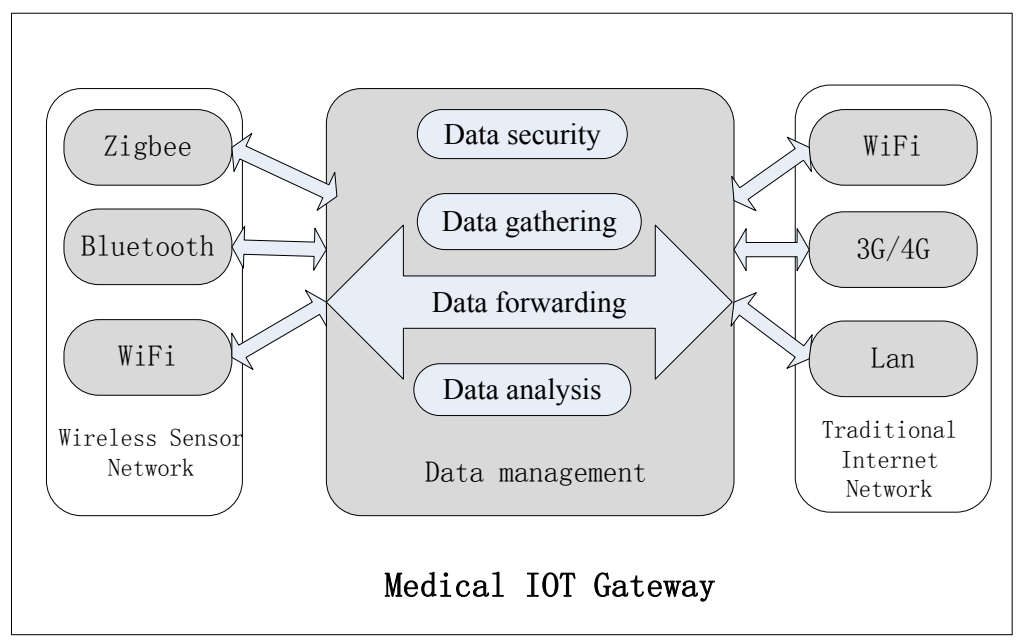

Fig. (2). Medical IOT gateway data management.

\subsection{Hardware Gateway Protocol Definition}

In hardware gateway, protocol conversion control layer provides protocol conversion from perception layer to the network layer. Data from the sensing devices is packaged in a standard unified data format which can be recognized by management platform (physiological parameter Gateway) management protocol; therefore, while hardware gateway translates data and controls commands, they can be recognized in sensor layer.

In wireless thermometer system, the nRF905 chip, which is a low-power consumption device, is embedded to carry the wireless transmission. The length of internal data transmitting registers of nRF905 chip is only 32 bytes, while address sending register is only 4 bytes long. Address sending register and data transmitting registers are independent. As the transmission is started, address information and data information is formed into a packet to be transmitted $[7,8]$. The data of package, including the physiological parameter, the value of physiological parameters, address information etc. will be unpacked at the hardware gateway. Physiological parameters include blood pressure (01), temperature (02), blood oxygen (03), pulse (04), ECG data (05) etc. nRF905 chip registers the packaging address and data information of the sending address register (TX_ADDR) and transmitting data register (TX_PAYLOAD), it sends the add prefix and CRC check code before starting the delivery mode to ensure the reliability of communication. So, we define the data packet form sent by the terminal device embedded with nRF905. Basic format is shown in the following Table 1:

Table 1. The terminal transmits data format.

\begin{tabular}{|c|c|c|c|c|}
\hline $\begin{array}{c}\text { Start } \\
\text { character }\end{array}$ & $\begin{array}{c}\text { Physiological } \\
\text { parameters }\end{array}$ & $\begin{array}{c}\text { Physiological } \\
\text { parameter } \\
\text { values }\end{array}$ & $\begin{array}{c}\text { The } \\
\text { address }\end{array}$ & $\begin{array}{c}\text { End } \\
\text { character }\end{array}$ \\
\hline
\end{tabular}

\subsection{The Terminal Transmits Data Format}

The Start character is defined as two bytes such as: 0Xaa, $0 \mathrm{Xaa}$, and End character is defined as 0X55, 0X55. The both characters will be judged at the hardware gateway first. As long as the two characters are all correct, the gateway will guarantee the correctness of the data transmission. This is just the format of data which is sent from terminal to hardware gateway. Hardware gateway need to send data to thermometer terminal as well. Hardware gateway acquires address information of the terminal after accepting data, and writes the address information into its address sending register (TX_ADDR). The data to be sent mainly includes two parts; one is ACK, another is Operating instruction, which is mainly used to require resending or modifying the address of the terminal. In such case, each thermometer works as a terminal, when sometimes the address of the terminal is needed to be modified it corresponds to bed number. The main data format is showed as following Table 2 .

Table 2. The data format of hardware gateway sending to terminal.

\begin{tabular}{|c|c|c|c|c|}
\hline $\begin{array}{c}\text { Start char- } \\
\text { acter }\end{array}$ & ACK & $\begin{array}{c}\text { Operating } \\
\text { instructions }\end{array}$ & $\begin{array}{c}\text { Information } \\
\text { of data }\end{array}$ & $\begin{array}{c}\text { End } \\
\text { character }\end{array}$ \\
\hline
\end{tabular}

\subsection{The Data Format of Hardware Gateway Sending to Terminal}

The Start character and End character are 0Xaa and 0X55 respectively. ACK is a set of confirmation instructions. Operation instructions message includes Modify address, Shutdown command and Resend instructions etc. Terminal address information is stored in the flash of MSP430 chip, so it is easy to call address information, or modify the address information through the instruction.

The data received by hardware gateway will be sent to the nurse station or physiological parameters gateway server through Wi-Fi without any modification, and the data transmission is easier than that is handled on the IPV4 network.

\subsection{Hardware Gateway Anti-Collision Control}

IOT gateway is the bridge to link wireless IOT and traditional WAN and LAN, and It faces a critical problem which is to route efficiently and manage the gateway stably and 


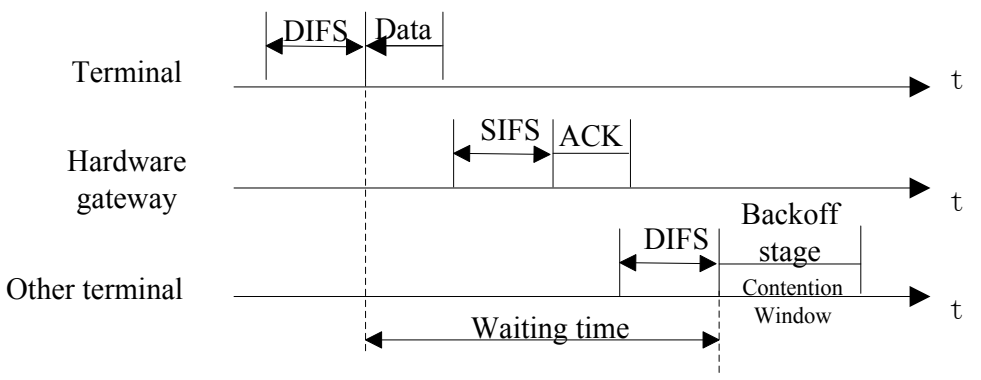

Fig. (3). CSMA/CA the basic operating principle.

reliably when large number of wireless terminal sensors link to the gateway simultaneously. The first problem solved is how to avoid transmission conflicts. In wireless thermometer system, low-power nRF905 chip is embedded with a transmission and communication protocol similar to CSMA/CA protocol, which is named after carrier sensing multipoint access with Collision Avoidance. As shown in Fig. (3), the protocol is defined as a kind of MAC protocol in the IEEE802.11 protocol [9].

When the terminal transmits data to the hardware gateway, channel carrier monitoring will be carried out before sending. The nRF905 chip has a function named Carrier Detect (CD). When the monitoring channel idle time is larger than Distributed Inter-frame Spacing (DIFS), the channel can be considered in idle status. If the channels are occupied during the monitoring time, the monitor will be operated continuously until the channel becomes idle. When a certain terminal finishes communication, the hardware gateway will release the channel soon. Then, the probability of conflict is the highest, because several terminals are waiting for the available channel to send data at that time. Therefore, when the idle channel is detected, terminals cannot send data immediately. The "binary exponential back off algorithm" is used to set the terminals on waiting. It is a period of back off time. The back off counter will start and decrease when the terminal monitor is in the idle channel. If it is detected that the channels are occupied, the back off counter will be suspended to maintain the value of the counter to be unchanged. When the idle channel is detected again, the back off counter decreases from the original foundation count. And it will start sending data when the count is reduced to zero. When the hardware gateway receives a data message, it waits for a Short interframe space (SIFS) and returns an ACK confirmation to the terminal.

There is a Carrier Detect pins CD in the wireless nRF905 chip. When the presence of carrier emits the same frequency in the air, the pin output level is high, and this feature is very effective to avoid the collision from different transmitters operating at the same frequency. As soon as the device is ready to transmit data, the chip is set into the reception mode, judging whether the frequency point of the channel is idle.

Function to achieve

$\operatorname{RecMod}() ; \quad / / S e t$ the receiving mode

while $((\mathrm{P} 3 \mathrm{IN} \& \mathrm{CD})==1)$; //Carrier monitoring

In CSMA/CA, in addition to the Carrier Detect, another aspect is to avoid the conflict. Delay stochastic time of Bina- ry back off algorithm is adopted to reduce the signal conflict probability to minimum, which improves the transmission success rate in order to improve the valuable transmission rate of channel. When a terminal in a wireless network is ready to transmit a packet, firstly it should determine a back off time initial (BI). The value is between $(0, \mathrm{CW})$, which is randomly selected in the range of the value interval, and $\mathrm{CW}$ is called "the contention window (Contention Window)". Binary back off algorithm features the CW size which depends on the number of times of collision experienced by sending data packets. Before the $\mathrm{CW}$ has not yet reached the maximum $\mathrm{CW}$, sending failure will lead to increased competition for the window every time. For example, the back off for $\mathrm{K}$ times is randomly selected in $2(2+\mathrm{k})$ slots. The maximum $\mathrm{CW}$ will be doubled. $\mathrm{BI}$ is uniformly distributed in the interval $(0, \mathrm{CW})$. So if $\mathrm{CW}$ is larger, back off time that the terminal randomly chooses may be longer and access channel is slower. The binary exponential back off algorithm can be expressed by the following formula:

$$
\left\{\begin{array}{l}
C W \leftarrow \min \left(C W \times 2, B_{\max }\right), \text { conflict } \\
C W \leftarrow B_{\min }, \text { successful transmission }
\end{array}\right.
$$

Back off time $=\mathrm{INT}[\mathrm{CW} * \text { Random }()]^{*}$ time gap

In the formula, Random() represents a pseudo-random number between $0 \sim 1$; time interval is for the characteristic value of physical layer. Implementation of $\mathrm{K}$ is based on the number of the conflict detection to determine the random backoff delay in the program. In order to avoid conflict, take random intervals in $2(2+\mathrm{k})$ slots. In the program, the embodiment of a delayed time is associated with the $\mathrm{K}$.

The basic implementation of the code is as follows:

Unsigned intrand_i $=0$;

while $((\mathrm{P} 3 \mathrm{IN} \& \mathrm{CD})==1) \quad / /$ Monitor whether have signal carrier

\{

rand_i $\mathrm{i}++$;

rand $j=\operatorname{rand}() \%\left(2^{\wedge}(2+\right.$ rand $\left.i)\right) ; / /$ To generate a random number rand $j$

//between $0-2^{\wedge}(2+\mathrm{k})$

delay(rand j j); //The delay of rand $j$ slots

if(rand_i $>$ max $)$ break; //More than the number of collisions

\} 
rand_i $=0 ; / /$ Reset rand_i

Where the function rand() is used to generate the random number and max is defined as the maximum collision time.

However, the hidden terminal problem affects the CSMA/CA, which exists in our system as well. The wireless nRF905 chips perform in half duplex, and each terminal has its own address respectively. For the address is special for the initialization of writing terminal, the data packets could be accepted effectively with a perfect address matching, while incorrect address can make the data unreadable, so it does not support the general radio. It doesn't have the information of occupied channel for each terminal, which might affect the realization of RTS/CTS and therefore, a handshake mechanism will not be considered. While the transmitted data quantity in this project is not large, so this problem will not cause a great loss of system. The hardware gateway can't receive the correct data because of the apparent conflict, and then it will be a failure to return the ACK signal to the terminal. As a result of deficiency of the confirmation message, the terminal will force the other terminal to send the fault state again for a reliable communication. Besides, the conflict probability will be relatively low because of the limited amount of data [7-8].

The sending and receiving chip configuration model is coded in $\mathrm{C}$ language as follows:

voidTransRF(void)

\{

P3OUT|=PWR_UP; //Start

P2OUT $\mid=$ TX_EN; //Transmit enable

TransTX_ADDRESS(); //Write to send address information mation

TransTX_PAYLOAD(); //Write to transmit data infor-

$$
\text { P3OUT } \mid=\text { TRX_CE; //Send start }
$$

$\operatorname{Delay}(100)$;

P3OUT\&= TRX_CE;

\}

Void RecMod()

\{

$$
\begin{aligned}
& \text { P3OUT } \mid=\text { PWR_UP; // Start } \\
& \text { P3OUT } \mid=\text { TRX_CE; //Send and receive enable } \\
& \text { P2OUT\&= TX_EN; //Send not to }
\end{aligned}
$$

\}

The main flow chart of the terminal such as clinical thermometer is shown in Fig. (4).

The transmitted data packets refer to transmitting data Frameworks. Every terminal has its own ID address, which can access the network at any time. The network is a kind of a radio network, so there is no accessing problem in the network. With the research mentioned above, we have carried out the first phase of design, wireless electronic thermometer system, which has been used in clinical practice. Wireless electronic thermometer is shown in the Fig. (5). Hardware gateway is shown in the Fig. (6).

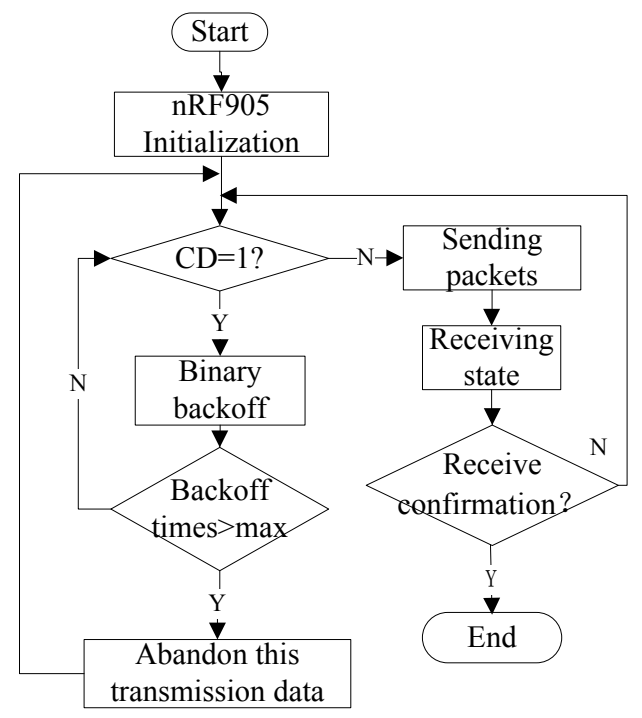

Fig. (4). The main flowchart of terminal operation.

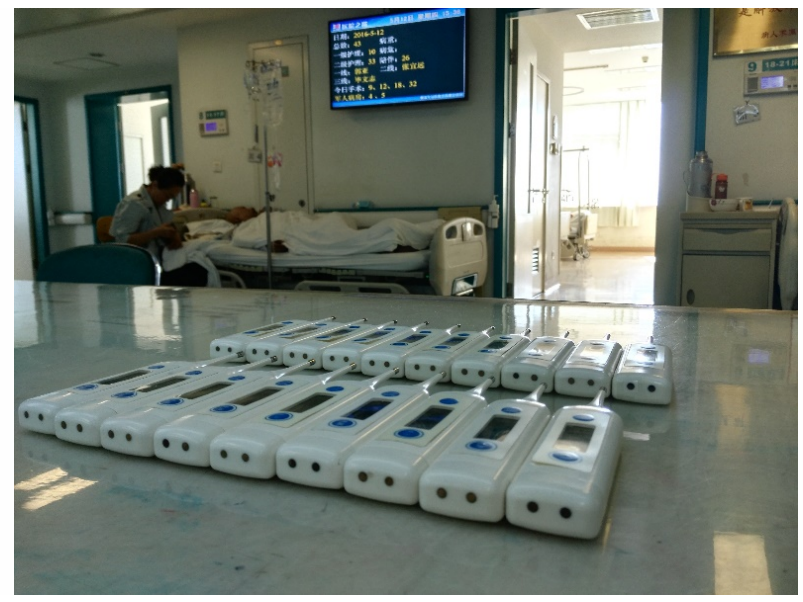

Fig. (5). Wireless electronic thermometer.

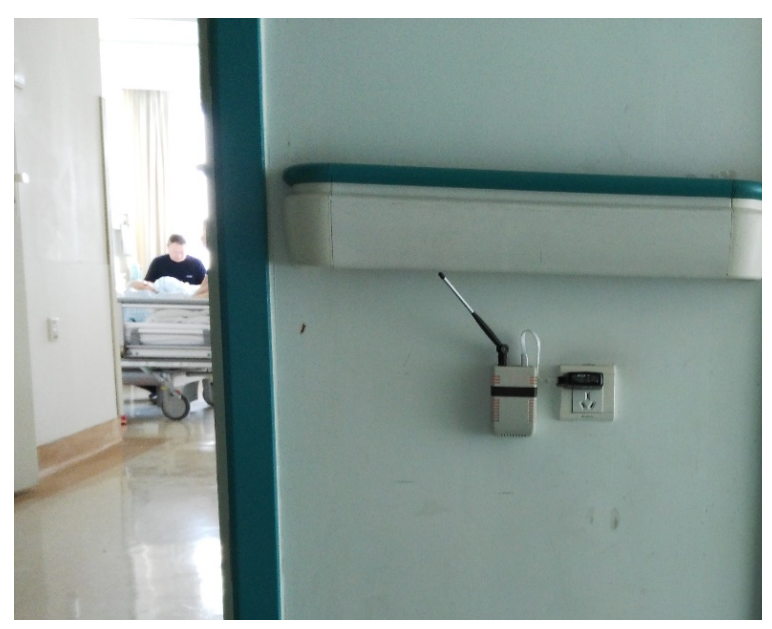

Fig. (6). Hardware gateway. 


\subsection{Real-Time High-Volume Data Forwarding}

In the process of monitoring physiological parameters, physiological parameters gateway has three main functions, 1) physiological parameter data forwarding, 2) monitoring hardware gateway accessing, 3) hardware fault monitoring. In the design process, the first task is a high-performance, real-time data forwarding. Such as, in the case of continuous ECG and PhotoPlethysmoGraphy (PPG) monitoring, we need the data forwarding technology to send the physiological data from the IOT devices to physiological parameters gateway, then copy to the nurses' station for medical purposes. During the monitoring ECG and PPG case, it is necessary to abstract requirements into a scientific problem and design a data copy mechanism to deal with the one to many and many to one data forwarding. In order to achieve such a goal, data forwarding mechanism with high performance is needed [10-12]. In this study, multi-thread programming based on $\mathrm{C}++$ Winsock is adopted, and the key point of the solution is that independent thread for service data acquisition is established for each connection so that each computer on the network can apply to view real-time monitoring data through the client application.

In the design of the gateway, performance cost of the server is the main consideration. Independent thread is occupied for each data distribution job. The service codes of independent thread are shown as below:

DWORD CWinsockTestDlg::memRecThreadClient (LPVOID lpParameter)

\{

memset(RecBuffer, 0x00, sizeof(RecBuffer));

len $=\operatorname{recv}($ ClientSocket- $>$ sock, RecBuffer, MAX_BUFFER_SIZE, 0);

if(!memcmp(RecBuffer, FrameHeadClient, 4))

//Judging whether the frame head received is consistent with the agreement

\{

bednum $=$ (unsigned char)RecBuffer[6] $* 256+$ (unsigned char)RecBuffer[7];

if $($ ClientSocket $->$ saveID $==0)$

//Judging whether it is the first time to receive the information of the terminal

\{

ClientArray[ClientCount*2] = RecBuffer[6];

//Put the bed number into the terminal array

ClientArray[ClientCount*2+1] = RecBuffer[7];

ClientSocket->bednum $[0]=$ RecBuffer $[6]$;

//Put the bed number into the Socket array of the terminal

ClientSocket- $>$ bednum[1] = RecBuffer[7];

ClientCount++;

ClientSocket- $>$ ID $=$ ClientCount;

ClientSocket->saveID $=1$;

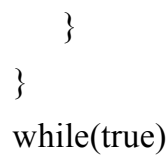

memcpy((ClientArray+i-2),(ClientArray $+\mathrm{i})$, entCount-ClientSocket->ID)*2); //Remove the terminal from the array

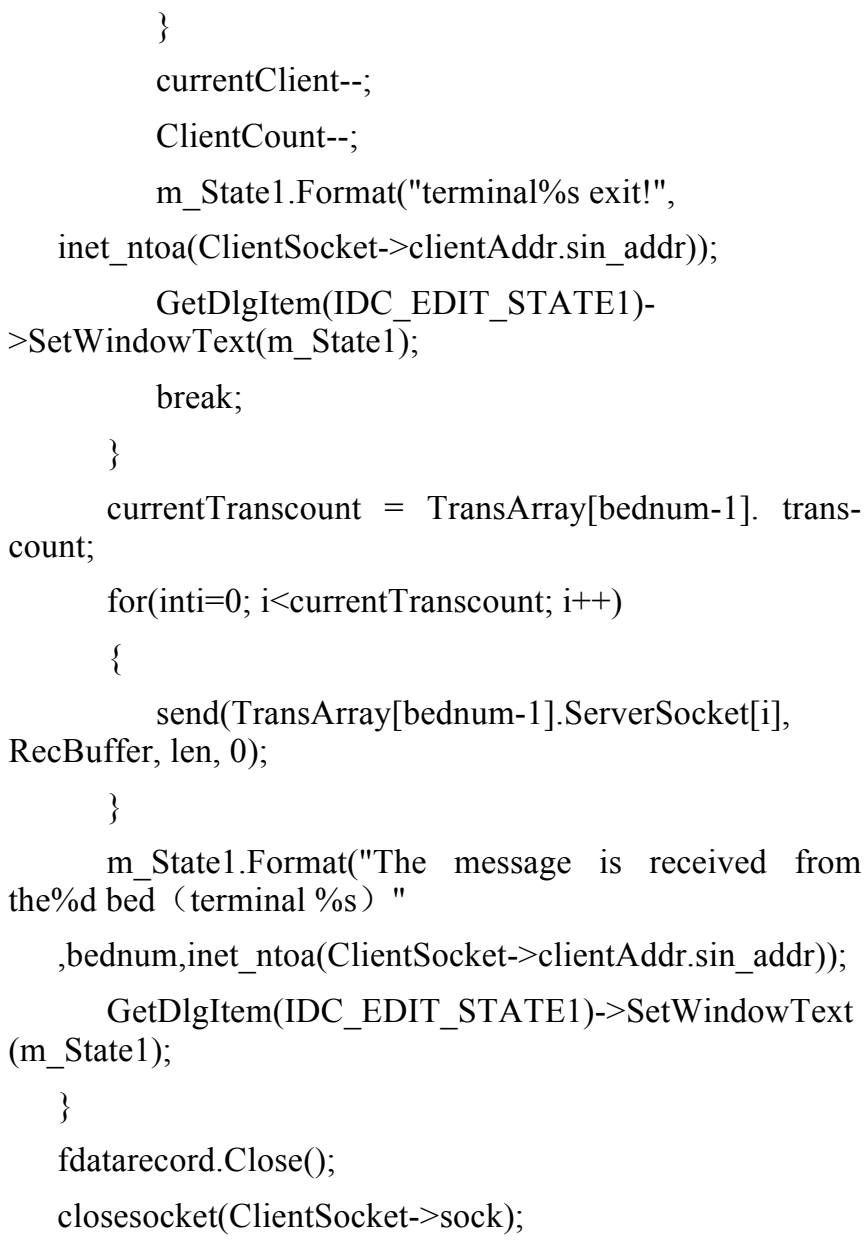

\subsection{Management of Hardware Gateway and On-Line Gateway Monitoring}

Hardware gateway receives data from the terminal and transmits it to the physiological parameters gateway continuously. Although numerous gateway hardwares are usually installed in different units, it is unrealistic for the personnel to maintain hardware gateway. Therefore, a real-time moni- 
toring is required to ensure that the gateway with low-power consumption works normally. In the design of mechanism of fault monitoring, an active submitting mechanism was adopted to report the fault state. The physiological parameter gateway checks the time intervals of receiving data from the hardware gateway. If a hardware gateway does not send data within the prescribed time, it will be identified to be in a temporary fault state, and the frequency of temporary failure will be counted by the physiological parameter gateway.

As soon as the data is received during the time interval, the temporary fault state will be canceled and the temporary failure count will be set to 0 . If the temporary fault state is detected for three times consecutively, the hardware gateway will be identified to be in fault state, and an alert message will be sent to the manager personnel. The workflow of the management system is shown in Fig. (7).

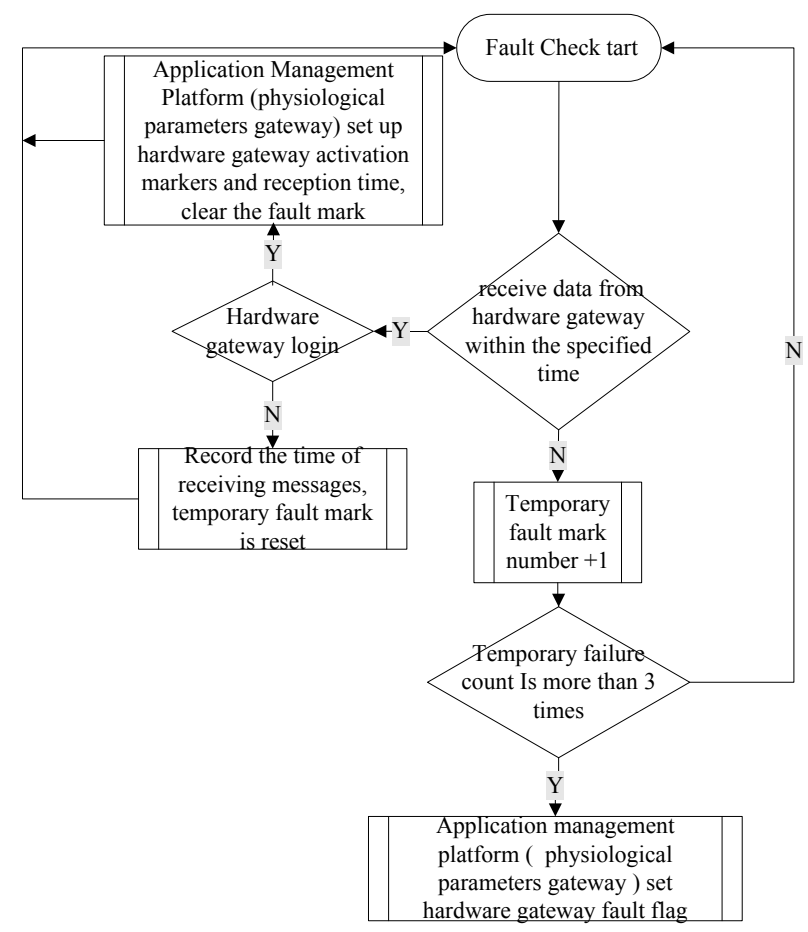

\section{CONFLICT OF INTEREST}

The authors confirm that this article content has no conflict of interest.

\section{ACKNOWLEDGEMENTS}

This work is supported by 2013 scientific research project of China National major projects (2013ZX03005012).

\section{REFERENCES}

[1] Q. M. Cheng, W. Pu, Y. B. Zhou, F. Wu, and M.S. Yu, "Dynamic monitoring system for clinical medical signals from area wards,"Chinese Medical Equipment Journal, vol. 29, no. 9, pp. 1214, 2008.

[2] J. Gao, W. Li, X. Feng, M.S. Yu, "Design and applied analysis of solutions for gateways and sensor nodes of medical internet of things," Chinese Medical Equipment Journal, vol. 8, pp. 41-45, 2014.

[3] Y.N. Hao, and Z.L. Hong," A Low-power long-range wireless data transmission platform based on MCU and nRF905 chip," Application of Electronic Technique, vol. 8, pp. 44-47, 2007.

[4] B. Hu,. Research on universal data acquisition and design and implementation of rule based data forwarding. Jilin University, 2006.

[5] Y.L. Li, S.F. Mo, "Novel query-driven real-time data forwarding in internet of things," Chinese Journal of Computers, 03: 464-476, 2012.

[6] L. Luo, "Design and implementation of wireless medical data acquisition system," Beijing: Beijing University of Posts and Telecommunications, 2011.

[7] H. Qi, Z. Xu, and H .Chen, "Design of short distance wireless data transmission system based on nRF905," Journal of Fuzhou University (Natural Science Edition), vol. 01, pp. 4-68, 2001.

[8] T.L. Shen, S. J. Hi, and Z.Z. Yan, "Design of the wireless medical information system based on PDA," Chinese Journal of Medical Instrumentation, vol. 31, no. 5, pp. 344-347, 2007.

[9] E.F. Wang, "Research on wireless sensor network MAC layer CSMA/CA mechanisms," Beijing University of Posts and Telecommunications, 2013.

[10] A.P. Xiao, "Development of an intellectualized nursing \& monitoring system in hospital wards," Chinese Journal of Medical Instrumentation, vol. 31, no. 2, pp. 36-39, 2007.

[11] Y.Q. Zhang and J.B. Li, "Design and implementation of doctorpatient management system based on IOT," Computer Study, vol. 1, no. 3, pp. 87-90, 2011.

[12] Y. Zhao, "Research based on the algorithm of sensor network data forwarding," University of Electronic Science and technology, 2005.

Fig. (7). The workflow of management system.

Received: May 26, 2015

Revised: July 14, 2015

Accepted: August 10, 2015

(C) Shi-lin et al.; Licensee Bentham Open.

This is an open access article licensed under the terms of the (https://creativecommons.org/licenses/by/4.0/legalcode), which permits unrestricted, noncommercial use, distribution and reproduction in any medium, provided the work is properly cited. 